\title{
Keratomycosis aspergillina als Ursache von
}

\section{Hypopyonkeratitis.}

\author{
Von
}

Professor Th. Leber in Göttingen.

Durch die Erfahrungen der jüngsten Zeit, insbesondere durch zahlreiche Versuche an Thieren ist die Thatsache sichergestellt, dass verschiedene Arten von Spaltpilzen (Schizomyceten) in der lebenden Hornhaut zu wachsen im Stande sind und dass ihr Wachsthum eine schwere eitrige Entzündung derselben hervorruft. Dagegen fehlt uns meines Wissens noch vollständig die Kenntniss, wie sich die höher stehenden Pilzarten, insbesondere die Schimmelpilze, in dieser Beziehung verhalten, wie überhaupt über die Möglichkeit des Wachsthums dieser Pilze in den verschiedenen Organen und Geweben des lebenden Körpers die Ausichten noch weit auseinandergehen.

Ich wurde veranlasst, mich eingehender mit dieser Frage zu beschäftigen durch einen einschlägigen Krankheitsfall, welchen ich, da er wohl der einzige dieser Art ist, welcher bisher beobachtet wurde, in den folgenden 
Zeilen etwas eingehender mittheilen und besprechen will. Es handelt sich um eine schwere Hypopyonkeratitis beim Menschen, bei welcher auf und in der Hornhaut eine sehr reichliche Entwickelung des Myceliums von Aspergillus glaucus gefunden wurde, welche ich nach dem Ergebniss einer grossen Zahl von Versuchen an Thieren als Ursache der eitrigen Keratitis betrachten muss. Da diese Versuche, welche bereits zu manchen interessanten Resultaten geführt haben, noch nicht $a b-$ geschlossen sind und später im Zusammenhang veröffentlicht werden sollen, so beschränke ich mich jetzt darauf, den betreffenden Krankheitsfall mitzutheilen und daran nur kurz die Resultate der damit angestellten Kulturversuche und derjenigen Experimente an Thieren anzuknüpfen, welche darthun, dass die Schimmelpilze wirklich in der gesunden lebenden Hornhant wachsen und Ursache von schwerer Entzündung derselben werden können.

\section{Krankengeschichte.}

Der Kranke war ein 54,jähriger sonst gesunder Landmann, welcher vor 5 Tagen beim Arbeiten mit der Dreschmaschine eine leichte Verletzung des Auges, wie er glaubt durch eine gegen dasselbe gepflogene Haferspelze, erlitten hatte. Schon am Tage darauf stellten sich Schmerzen im Auge ein, welche sich an den folgenden Tagen zu ungewöhnlicher Heftigkeit steigerten, während zugleich das Sehrermögen stetig abnahm. $\mathrm{Da}$ die anderwärts verordnete Atropinlösung keine Besserung hervorrief, suchte der Kranke am 26. MIärz d. J. in der Augenklinik Hülfe.

Ich fand ein Hornhautgeschwür von 4-5 Mm. Durchmesser, welches sich von der Mitte nach dem lateralen Rande der Hornhaut hin ausdehnte, mit weissgrau getrübtem Grund und leicht aufgeworfenem Rand, ein Hypopyon, welches bis zum unteren Rande des Geschwüres reichte und ausgesprochene Chemosis der Scleralbindehaut. Die Pupille liess sich durch Atropin noch gut erweitern. Wegen des centralen Sitzes des Geschwürs wurden nur Finger in nächster Nähe gezühlt. Ein 
Thränensackleiden war nicht rorhanden; der Kranke hatte nie an Thränenträufeln gelitten, auch liess sich weder jetzt noch während des ganzen weiteren Verlaufes jemals eine Spur von Secret aus dem Thränensack ausdrücken.

Aufnahme in die Klinik und Behandlung mit Schutzverband und Einträufelungen von Atropin und von Aqua Chlori abwechselnd.

Anfangs trat hierauf einige Besserung ein; das Geschwür schien stehen zu bleiben, das Hypopyon nahm nicht $z u$ und der Kranke befand sich ohne orhebliche Schmerzen. Nach zwei Tagen veranlasste das Wiederanftreten stärkerer Schmerzen und ein geringes Weiterschreiten des Geschwürs bei etwas stärkerer Chemosis zu einem Versuch mit warmen Umschlägen. Nachdem aber der Kranke in der Nacht rom 29. zum 30. März wieder viel Schmerzen gehabt, auch das Hornhautgeschwür sich nasalwärts noch etwas vergrössert hatte, so entschloss ich mich am 30. Mărz zur Querspaltung nach Sämisch's Methode. Der Pupillarrand war noch frei, anch das Hypopyon nicht besonders vergrössert, die Chemosis bedentend. Die Prognose wurde zweifelhaft gestellt, weil ich starke Chomosis in früheren Fällen als ungünstiges Zeichen kennen gelernt hatte.

Das innere Ende des Schnittes fiel wegen starken Kneifens der Lider von Seiten des Kranken gerade an den inneren Geschwürsrand, ohne ihn, wie es sein sollte, noch etwas zu überragen, das in der Wunde liegende geronnene Hypopyon liess sich aber mit der Pincette leicht und vollständig entfernen, und die Kammer war darauf vollständig frei. Einträufelung von 1 proc. Carbolsäurelösung und Borverband.

Am folgenden Tag (31. März) hatte sich das Geschwür trotz regelmässiger und sorgfältiger Wiederöffnung der Wunde besonders nach oben hin noch stetig vergrössert; doch erschien die Chemosis geringer und das Hypopyon nicht regenerirt; anch hatten sich die Schmerzen wesentlich vermindert. Das Geschwür erreichte schon ziemlich den oberen Hornhantrand; von seinem oberen Rande schien sich eine weissgraue Membran ein wenig abzuheben, von welcher sich mit der Irispincette leicht ein Kleines Fetzchen fassen und abziehen liess. Ich erinnerte mich jetat auch, dass schon von Anfang an der Geschwürsgrund von einer ähnlichen weissgrauen Schicht bedeckt schien. Dies veranlasste mich zur mikroskopischen 
Untersuchung des kleinen, nur 2-3 Mm. grossen Stückchens. Ich brachte dasselbe direct von der Pincette auf einen Objectträger und fand es zu meinem Erstannen dicht durchsetzt von Fadenpilzen.

Man konnte daran deutlich zwei über einander liegende Schichten unterscheiden; in der eịnen lagen schmalere und mehr gestreckte, baumförmig verästelte und gegliederte Pilzfäden von dem Aussehen des Myceliums gewöhnlicher Schimmelpilze, eingebettet in eine trübe, feinkörnige Masse, welche sich an der namentlich beim Zerzupfen hervortretenden fibrillären Beschaffenheit als necrotische Hornhautgrundsubstanz zu erkennen gab. Letztere enthielt stellenweise neben den Pilzen auch mobr oder minder zahlreiche Eiterkörperchen eingelagert; daneben fanden sich noch kleine .Stäckchen zum Theil veränderten Epithels oder einzelne Epithelzellen.

Die andere Schicht zeigte breitere, stärker lichtbrechende, viel mehr and unregelmässiger verzweigte Pilzfäden, welche dicht zusammengedrängt und oft auf das innigste durcheinander gewirt und geflochten waren und dadurch ein sehr eigenthümliches Bild darboten. Die einzelnen Elemente traten bei ihrer diebten Aneinanderlagerung und Einbettung in das auch hier feinkörnig getrübte necrotische Hornhautgewebe nicht sehr klar hervor und nur an Zupfpräparaten gelang es, sich über das Vorhalten einigermassen zu orientiren. Die Pilzfäden waren breiter, deutlich doppelt contourirt und glänzend und erhielten durch sehr zahlreiche, aber meist kurze Sprossen eine sehr unregelmässige, vielfach ausgebuchtete Begrenzung. Die nach oben und unten abgehenden Seitenäste ersehienen dabei im optischen Querschnitt als glänzende, der Faser scheinbar auf- oder eingelagerte Ringe. Debrigens handelte es sich hier keineswegs um zwei versehiedene Pilzarten, denn man konnte wiederholt sich mit Bestimmtheit überzeugen, dass die beiden Formen von einer Sehicht zur anderen in unmittelbarem Zusammenhange standen.

Zu erwahnen ist noch, dass die isolirten Pilzfaden sich vielfach von einem hellen Saum umgeben zeigten, welcher sich nach aussen an die Zellmembran anschloss, nicht sehr scharf und gleichmässig begrenzt erschien und besonders bei Tinction deutlicher hervortrat.

Bei der frischen Untersuchung glaubte ich das feinkörnige Aussehen der Grundsubstanz zum Theil auf gleichzeitige Ein- 
lagerung von Microcokken beziehen zu müssen, obwohl die Körnchen durch Hämatoxylin keine stärkere Färbung annahmen; bei nochmaliger Durehsicht der unterdessen in Clyeerin aufbewahrten Präparate, welche sich ganz gut gehalten und noch an Klarheit gewonnen haben, ist aber das feinkörnige Aussehen ziemlich verschwunden und es lässt sich von Spaltpilzen durchaus nichts erkennen. Es ist mir deshalb zweifelhaft geworden, ob auf der Hornhaut überhaupt Microcokken vorhanden waren und ich möchte annehmen, dass sie, wenn überhaupt, jedenfalls nur in geringer Menge, vielleicht nux in den schon ganz im Zerfall begriffenen oberflächlichen Schichten vorkamen.

Welche von den beiden Schichten die oberflächliche und welche die tiefe war, liess sich direct nicht mehr bestimmen, doch möchte ich vermuthen, dass die vielfach verästelten und durch einander gewirrten, breiten Pilzelemente mehr den tieferen Schichten des Hornhautgewebes angehörten, während die schmaleren Pilzfäden von dem gewöhnlichen Aussehen sich wohl mohr an der Oberfläche entwickelt haben mochten, wo sie normalere Lebensbedingungen finden mussten. Doch ist dies nur eine Vermuthung und ich habe auch bei meinen Versuchen an Thieren ein gleiches Verhalten nicht beobachtet. Unser Botaniker, Prof. Reincke, welcher die Freundlichkeit hatte, meine Präparate anzusehen, konnte zunächst bestätigen, dass es sich um ein Pilzmycelium handelte, und gab mir den Rath, da in Ermangelung von Fructificationen eine botanische Bestimmung derselben vorläufig nicht ausführbar war, Culturversuche anzustellen, über deren Resultat ich weiter unten berichten werde.

Ich bemerke schon hier, dass die Pilze zweifellos sich während des Lebens in der Hornhaut entwickelt haben mussten, da ich das von der Hornhant abgezogene Partikelchen sofort und ohne Zusatz auf einen Objectträger brachte und mikroskopisch untersuchte.

Nach Reinigung der Geschwürsfläche von den noch anhaftenden Resten der Auflagerung wurde dieselbe vorsichtig mit 5 proc. Carbolsäurelösung gepinselt und mehrmals täglich 1 procentige Carbolsäurelösung in den Conjunctivalsack eingeträufelt.

Am 1. April hatte sich das Geschwür besonders nach unten hin noch weiter vergrössert, die Ränder des Hornhant-

v. Graefe's Archiv für Ophthalmologie, XXV. 2. 
schnittes waren von einer graulichen Substanz leicht auseinander gedrängt, welche ebenso wie der wiederum entfernbare geringe Belag des Geschwürsgrundes reichliche Mengen derselben Pilze enthielt. Die Wiedereröffnung der Wunde mit der Sonde wurde deshalb jetzt als nutzlos aufgegeben. Um kein Mittel unversucht zu lassen, liess ich eine Lösung von schwefliger Sänre anfertigen, mit welcher das Geschwür 2 bis 3 Mal täglich bepinselt und ein Wenig davon in den Conjunctivalsack eingeträufelt wurde, da die schweflige Säure gerade für Fadenpilze als das wirksamste Gegenmittel betrachtet wird. Doch gelang es damit eben so wenig, dem schon zu weit gediehenen Wacherungsprocesse Einhalt zu thun.

Die Hornbaut war nach zwei Tagen, am 3. April, bereits total getrübt und ulcerirt, weshalb am darauf folgenden Tage dies Mittel, welches jedes Mal heftiges Brennen für etwa eine Stunde lang hervorrief und während dessen Gebrauch eine etwas stärkere Absonderung der Conjunctiva entstanden war, ebenfalls ausgesetzt wurde. Nur der Umstand könnte vielleicht für die Wirkung der schwefligen Säure sprechen, dass ein Kulturversuch mit Pilzen, welche während ihrer Anwendung von der Hornhaut gewonnen waren, kein Resultat lieforte, während spätere Kulturen, auf dieselbe Weise vorgenommen, leicht gelangen.

Am 5. April erschien die Hornhaut total grauweiss getrübt, an ihrem oberen Rande eine schmale Rinne, deren peripherer Rand von dem etwas mehr vascularisirten Limbus corneae, der centrale von einer in der Ablösung begriffenen grauweissen Masse gebildet wurde, von welcher sich aber nur einige ganz kleine Partikelchen abheben liessen; letztere enthielten wioder zahlreiche Pilzelemente. Nach unten zu schien ein wenig eitriges Exsudat aus der vorderen Kammer durchzusehimmern. Im weiteren Verlauf breitete sich die erwähnte Rinne über den ganzen Hornhautrand aus und die Auflagerung des Geschwürsgrundes stioss sich allmälig ab; die zu wiederholten Malen vorgenommene mikroskopische Untersuchung wies bis uum 11. April noch regelmässig in den mit der Pincette bald mehr bald weniger leicht entfernbaren Fetzchen dieselben Pilze wie früher nach. Unterdessen hatte sich die Hornhaut sehon ein wenig verkleinert and ibre Oberfläche gereinigt, die Ränder des Schnittes waren aber noch immer von etwas graulicher Zwischensubstanz auseinander- 
gedrängt. Durch Einträuflungen einer schwachen Zinklösung hatte sich der Conjunctivalkatarrh verloren und der Patient wurde daher am 15. April in voller Reparation, mit Aussicht auf Bildung eines Leucoma totale entlassen, mit welchem or sich auch einige Wochen später, nachdem der Process vollkommen abgelaufen war, wieder vorstellte.

Um die Pilze botanisch bestimmen zu können, stellte ich auf den Rath von Prof. Reincke einige Kulturversuche an, von welchen die in verdünntem und längere Zeit gekochtem Fruchtsaft das beste Resultat lieferten. Sie ergaben, dass es sich um einen der gewöhnlichen Schimmelpilze, und zwar um Aspergillus glaucus handelte.

Es wurden bei diesen Versuchen alle nöthigen Vorsichtsmassregeln beobachtet: Die Kultur geschah bei hängendem Tropfen auf einem Deckgläschen über einem hohl geschliffenen Objectträger, dessen Delle, um die Verdunstung zu verhäten, mit etwas zuvor gekochtem Wasser benetzt wurde, eine $\mathrm{Me}$ thode, bei welcher das etwaige Wachsthum der Pilze jeden Angenblick controlirt werden konnte; überdies wurde das Ganze mit einer reinen Glasglocke überdeckt; alle benützten Instrumente waren auf das sorgfaltigste gereinigt und unmittelbar vor dem Gebrauch an der Spirituslampe geglüht; der Rest der Kulturfüssigkeit blieb zur Controle unter Watteverschluss längere Zeit stehen, ohne dass sich Pilze darin entwickelten. Zar Aussaat wurde ein soeben dem lebenden Ange entnommenes Stückchen pilzhaltiger necrotischer Hornhautsubstanz benutzt, von welchem mit geglähten Nadeln einige möglichst feine Partikelchen abgelöst und rasch in einen Tropfen der Fruchtsaftlösung gebracht wurden. Dieselben schrumpften durch die Wirkung dieser saner reagirenden Flüssigkeit etwas zusammen und wurden vollständig opak, weshalb es nicht mehr wie an anderen eben so bleinen Partikelchen möglich war, die in sie eingeschlossenen Pilze mikroskopisch zu beobachten. Schon am nächsten Tage sah man aber an verschiedenen Stellen ihrer Oberfläche kurze Pilzfäden zum Vorschein kommen, welche sich an den folgenden 
Tagen mit grosser Geschwindigkeit verlängerten und vermehrten, so dass sehr bald jedes der kleinen Pünltchen mit mit der Loupe und später auch mit blossem Ange sich als Ausgangspunkt eines zarten und dichten Schimmelrasens erkennen liess. Die Pilzfäden wuchsen so dicht und gleichmässig von der ganzen Oberfläche jedes der kleinen Stückchen nach allen Seiten aus, dass schon deshalb an eine zufällige Verunreinigung nicht gedacht werden kann, abgesehen davon, dass dieselbe schon durch die Versuchsmethode sicher ausgesehlossen ist. Erst nachdem die Pilzfäden eine gewisse Länge erreicht hatten, fingen sie an, besonders in der Nähe ihrer Basis, seitliche Sprossen und Aeste zu treiben. Die freien Enden erschienen in grosser Ausdehnung ungetheilt; weiter zurück sah man an den Fäden zarte Querwände, durch welche sie in etwas ungleich lange, aber doch ziemlich lange Zellen zerfielen. Die Fäden enthielten zahlreiche kleine helle Tröpfichen von ungleichem Durchmesser, welche gegen das freie Ende des Pilzfadens immer kleiner wurden und zuletzt nicht mehr zu erkennen waren.

Bei ihrem Längenwachsthum erreichten die Enden der Pilzfäden bald die Grenze des Flüssigkeitstropfens; nach einiger Zeit sah man sie selbst noch etwas darüber hinaus ragen, indem jeder Pilzfaden von einem schmalen Flüssigkeitsstreifen auf beiden Seiten begleitet war, welcher ihm durch Capillarität adhärirte; vermuthlich war die dazwischen befindliche Flüssigkeit, deren Verdunstung ja durch die feuchte Kammer verhütet war, durch das rasche Wachsthum der Pilze verbraucht worden. Unterdessen kam es za einer reichlichen baumförmigen Verästelung der Făden nach allen Seiten hin, somit zur Bildung eines vollständig entwickelten Pilzmyceliums.

Sehr bald traten nun an den an die Luft grenzenden Finden der Pilzfäden Fructificationen auf in Gestalt von opaken, im durchfallenden Lichte schwarz aussehenden Köpfehen, welche, wie auch die letzten Enden der Fäden selbst, frei in die Luft hinanfragten; auch diese Enden der Fäden erschienen daher opak, mit breiten schwarzen Contouren versehen, einfach in Folge der mangelnden Benetzung und des grossen Unterschiedes im Brechungsindex zwischen Luft und Pilzmembran. 
In einem der Versuche wurden dieselben 5 Tage nach Beginn der Kultur auf dem Objectträger zuerst beobachtet.

Zur genaneren mikroskopischen Untersuchung dieser Fructificationen ist es nöthig, sie vorher zu benetzen, was mit Wasser nicht gut gelingt, aber leicht mit einem Tropfen einer Mischung von Alkohol, Glycerin und Wasser. An solchen Präparaten sah man nun sehr klar die charalkteristischen Conidienträger des Aspergillus; das Ende des Pilzfadens schwillt kolbenförmig an und die Anschwellung ist dicht besetzt mit kleinen stäbchenförmigen Zellen, sog. Sterigmen, an deren Ende eine Reihe von Sporen sitzen; Sterigmen und Sporen sind dicht an einander gedrängt, so dass daraus ein compaktes rundliches Köpfchen entsteht. Die grünliche Farbe der Sporen des Aspergillus glaucus war besonders auch an den ganzen Köpfchen bei auffallendem Lichte mit der Loupe sehr schön zu sehen, noch bequemer aber und mit blossem Auge bei später unter gleichen Cautelen angestellten Massenculturen in einem mit verdünntem Fruchtsaft gefüllten Kölbchen, bei welehen sich an der Oberflache ein dicker graugrüner Staub von Schimmelsporen entwickelte.

Das Ergebniss dieser Kulturversuche ist also, dass es sich um eine Art der Gattung Aspergillus, handelt, von welcher auch sonst schon mehrere Arten in verschiedenen anderen Organen des lebenden menschlichen Körpers angetroffen worden sind. Hauptsächlich ist hier zu erwähnen ihr Vorkommen in der Lunge (Pneumonomycosis aspergillina) und auf dem Trommelfell (Myringomycosis aspergillina). Letzteres hat offenbar die grösste Analogie mit dem in der Hornhaut und es ist daher von Interesse, die darüber bekannten Thatsachen zum Vergleich heranzuziehen. Ich folge dabei den Angaben von Wreden, der wohl die zahlreichsten und eingehendsten Untersuchungen über die Mycosis des Trommelfells angestellt hat*).

*) Vergl. R. Wreden: Die Myringomycosis aspergillina in den Jahren 1869-1873 nach eigenen und fremden Beobachtungen besprochen. Arch. f. Augen- u. Ohrenheilk. III. 2, S. 56-90. (1874). 
Mit wenigen Ausnahmen handelte es sich um Aspergillusarten, meist Asp. flavescens und nigricans, seltener Asp. glaucus. Die Pilze entwickeln sich nach Wreden nicht secundär auf einem vorher erkrankten Trommelfell, sondern selbständig und primär. Ihre Wucherung beschränkt sich in der Regel auf die Epidermis des Trommelfells und des angrenzenden Theiles des äusseren Gehörganges und ruft hier eine Entzündung von meist nur mässiger Intensität hervor, die gewöhnlich in Genesung ausgeht; in Ausnahmefällen aber dringt die Pilzwucherung in die tieferen Schichten des Trommelfells ein und kann durch eitrige Entzündung zur Perforation desselben führen, worauf die Pilze selbst auf die Schleimhaut der Paukenhöhle sich weiter verbreiten können.

Gegenüber der Anschaunng, dass die Schimmelpilze nicht die Rolle wahrer Parasiten spielen könnten, sondern immer nur die sogenannter Saprophyten, welche auf abgestorbenen Gewebstheilen wuchern, hebt Wreden bestimmt hervor, dass die Pilze in die Gewebe des gesunden Trommelfells hineinwachsen und von demselben nicht eher entfernt werden können, als bis die befallenen Schichten durch reactive Entzündung abgelöst sind. Die Richtigkeit dieser durch klinische Beobachtungen gewonnenen Ansicht wurde u. A. von Politzer durch anatomische Untersuchung eines derart erkrankten Trommelfelles direct bewiesen. Diese Feststellung ist deshalb von besonderer Wichtigkeit, weil an inneren Organen, wie z. B. in der Lunge, sich daräber weit schwerer genügende Sicherheit gewinnen lässt.

Auch in meinem Falle von der Hornhaut muss unstreitig die Pilzwucherung als das Primäre und die Ent. zündung als das Secundäre betrachtet werden.

Dass während des Aufenthaltes in der Klinik Schimmelpilze in der Menge, wie sie zur Beobachtung kamen, zufällig anf die Hornhaut gelangten, ist rein unmöglich; 
wollte man selbst die Möglichkeit zugeben, dass trotz der grössten Reinlichkeit einmal einige Schimmelpilze auf die Hornhautoberfläche gelangen konnten, so hätten doch bei den alle zwei Stunden wiederholten Einträufelungen und der zwei Mal täglich von mir oder von einem meiner Assistenten vorgenommenen sorgfältigen Reinigung des Auges von allen Secreten längst alle solche Fremdkörper wieder entfernt sein müssen; statt dessen liessen sich während 11 Tagen regelmässig dicht gedrängte Anhäufungen der Pilze von der Hornhaut entfernen, welche ihr nicht oberflächlich auflagen, sondern ganz unzweifelhaft in kleine Fetzen einer sich nach und nach abstossenden necrotischen Schicht der Hornhaut eingelagert waren. Da immer nur die schon ganz erweichten, lose anhaftenden Partien dieser Schicht mit Irispincette oder Daviel'schem Löffel vorsichtig entfernt wurden, konnte man sich bestimmt davon überzeugen, dass die pilzhaltigen Stückchen, welche an einem Tage entfernt wurden, am Tage zuvor noch in organischer Verbindung mit der Hornhaut gestanden hatten. Auch die mikroskopische Untersuchung, welche daran Spuren einer fibrillären Beschaffenheit erkennen liess, sowie das Aussehen für das blosse Auge und die Consistenz zeigten, dass die Grundsubstanz, in welche die Pilze eingebettet waren, wirklich necrotisches Hornhantgewebe war.

Es ist deshalb anzunehmen, dass die Aussaat dieser Schimmelbildung in das Hornhautgewebe schon vor der Aufnahme des Kranken in die Klinik erfolgte und es ist überhaupt wohl keine andere Möglichkeit denkbar, als dass sie bei der Verletzung selbst stattfand. Wenn man sich erinnert, dass schon am Tage nach der Verletzung Entzündung mit lebhaften Schmerzen begann, wobei doch sicher das Auge meist geschlossen, überdies wohl in der Regel mit einem Tuche verdeckt gehalten 
wurde, wo überdies das Anhaften etwaiger später trotzdem hinein gelangter fremder Substanzen durch die vermehrte Absonderung von Thränen and Bindehautsecret verhindert werden musste, so wird man gewiss zur Ueberzeugung kommen, dass nur bei der Verletzung selbst die Pilzkeime in die Cornea gelangt sein konnten. Bei der Art der Verletzung braucht man auch nach der Quelle der Pilze nicht weit zu suchen; da das im März gedroschene Getreide wahrscheinlich, wie es hier zu Lande üblich ist, von der Zeit der Ernte an za grossen Haufen aufgeschichtet im Freien gelegen hatte, so ist es kaum denkbar, dass sich in dieser Zeit auf den Hüllen der Körner oder auf den Strohhalmen nicht Schimmel in Menge entwickelt haben sollte.

Kana also die Frage, ob die Aspergilluspilze in dem hier vorliegenden Falle in der lebenden Hornhaut gewachsen seien, schon durch die klinische Beobachtung bejaht werden, so findet diese Annahme noch eine weitere zweifellose Bestätigung durch die Ergebnisse der von mir angestellten Versuche an Thieren. Durch fractionirte Kulturen gelang es, die Aspergilluspilze in beliebiger Menge vollkommen rein zu erhalten; mit diesem Material wurden dann zahlreiche Versuche an Kaninchen angestellt, deren Resultat sich kurz dahin zusammenfassen lasst, dass der Aspergillus glaucus in der lebenden Hornhant sich sehr rasch entwickelt and ein ungemein lebhaftes Wachsthum zeigt. Es war dafür einerlei, ob man ein kleỉnes Partikelchen des Pilzmyceliums in eine Hornhautwunde einführte oder eine indifferente Flüssigkeit, welche Aspergillussporen suspendirt enthielt, in das Hornhautgewebe einspritzte. Die Pilze wuchsen mit überraschender Geschwindigkeit der Fläche und Tiefe nach in die vorher ganz unveränderte Hornhaut hinein und zwar vollkommen unabhängig von den Structurverhältnissen des Hornhautgewebes. In 
einem Falle hatten sie z. B. schon nach 24 Stunden etwa $2 / 3$ der Hornhautdicke durchsetzt und sich der Fläche nach über einen etwa $5 \mathrm{Mm}$. im Durchmesser betragenden Abschnitt der Hornhaut verbreitet; nach zwei Tagen hatte sich dieser Heerd noch mehr vergrössert und es war bereits die ganze Dicke der Hornhaut durchwachsen.

Diese Versuche lieferten nun noch ein weiteres Ergebniss, welches durch die klinische Beobachtung allein nicht mit Sicherheit gewonnen werden konnte, dass nämlich das Wachsthum des Aspergillus in der lebenden Hornhaut eine ganz ähnliche Form von schwerer eitriger Keratitis hervorruft, wie wir sie als Folge der Infection mit Schizomyceten kennen. Bei der grossen Wichtigkeit, welche diese Beobachtung für die Entzündungslehre besitzt, muss ich mir die ausführlichere Mittheilung dieser Versuche, welche ich zur Zeit nach einigen Richtungen hin noch weiterführe, für später vorbehalten; ich kann aber schon jetzt versichern, dass diese Entzündung nach Injection vollkommen reiner Sporen von Aspergillus, welche in indifferenter, $3 / 4$ proc. Na Cl-lösung aufgeschwemmt waren, ganz constant mit voller Intensität und grosser Raschheit zur Entwickelung kam.

Wenn es somit feststeht, dass Fadenpilze, welche in der Hornhaut sich entwickeln, ebenso stark entzündungserregend wirken, als Schizomyceten, so folgt daraus doch für unseren Krankheitsfall noch nicht ohne Weiteres, dass sie daselbst die wirkliche und einzige Entzündungsursache gewesen seien. Die Verhältnisse sind hier vielmehr dadurch verwickelt, dass wahrscheinlich ausser dem Aspergillus auch Microcokken auf der Hornhaut des Kranken vorhanden oder wenigstens nicht mit Sicherheit auszuschliessen waren. Wie oben bemerkt, wurden in den von der Hornhaut entfernten necrotischen Gewebsfetzen $z$ wischen den Fadenpilzen 
feine Körnchen gefunden, welche ich anfangs zum Theil für Microcokken gehalten hatte, während mir späterhin deren Nachweis zweifelhaft wurde. Für ihre Anwesenheit sprach aber auch ein Kulturversuch, welchen ich mit dem direct der kranken Hornhaut entnommenen Material auf einem dünnen Schnitte von gekochter Hornhautsubstanz anstellte. Derselbe ergab zunächst nur Wucherungen von Microcokkenhaufen auf der Hornhautoberfläche und von beweglichen Bacterien in der umgebenden Flüssigkeit. Erst nach zwei Tagen wuchsen auch Aspergillusfäden in den Flüssigkeitstropfen hinein, aber noch nicht in das Hornhautgewebe; sie waren zum Theil mit einem zarten und dichten Rasen kurzer Bacterien überzogen, welche ihnen senkrecht zu ihrer Oberfläche ringsum dicht gedrängt aufsassen. Erst nach 6 Tagen waren die Aspergillusfäden auch in die Hornhaut hineingewachsen und verbreiteten sich in ihrer Substanz zwischen den sehr schön sichtbaren Hornhautkörperchen nach allen Richtungen hin. Solche mit Bacterien besetzte Pilzfäden, aber in geringerer Menge, fanden sich auch bei den ersten Kulturen in Fruchtsaft, verschwanden aber bei den späteren Reinkulturen vollständig.

Da der nicht zu dem genannten Versuch benutzte Rest der gekochten Hornhaut noch fün Tage später in einem mit Banmwolle verstopften Kölbchen mit ein wenig gekochtem Wasser sich ganz frisch und ohne jede Spur von Fäulniss erhalten hatte, so ist wohl anzunehmen, dass die bei dem Kulturversuch zur Entwickelung gekommenen Spaltpilze von Keimen abstammten, welche schon in dem Impfmaterial enthalten waren.

Wenn sich nun hieraus auch mit ziemlicher Wabrscheinlichkeit ergiebt, dass in dem mitgetheilten Falle 
auf der lebenden Hornhaut nicht nur Fadenpilze, sondern auch Microcokken zur Entwickelung gekommen waren, so folgt daraus doch noch Nichts für die Entstebungsursache der eitrigen Keratitis. War letztere zunächst allein durch die Aspergilluswucherung verursacht, wovon die Möglichkeit ja durch meine Versuche an Thieren sicher festgestellt ist, so konnten sich an der Hornhautoberfläche, wenn es daselbst schon zur Bildung eines Geschwürs gekommen war, späterhin auch Microcokken angesiedelt haben, welche dann ihrerseits zum weiteren Zerfall der Gewebe beitrugen.

Andererseits muss auch an die Möglichkeit gedacht werden, dass mit den Fadenpilzen sich gleich von Anfang an auch Spaltpilze auf der Hornhaut entwickelt batten, so dass alsdann beide gemeinschaftlich die Entzündung hervorgerufen hätten, während die dritte Annahme, dass die Aspergilluspilze erst nachträglich auf der bereits durch Wucherung von Microcokken in Entzündung versetzten Hornhaut gewachsen seien, aus den oben angeführten Gründen zurückgewiesen werden muss.

Obwohl nun eine sichere Entscheidung zwischen den beiden zuerst genannten Möglichkeiten nicht getroffen werden kann, so möchte ich doch die erste für die wahrscheinlichere halten. Bei gleichzeitiger Infection mit Aspergilluselementen und Microcokken würden wohl die letzteren, für welche die Hornhaut bekanntlich ebenfalls einen sehr günstigen Boden abgiebt, bald die Oberhand erhalten haben, wie wir dies auch sonst bei alkalischer oder neutraler Reaction des Nährbodens zu beobachten pflegen, und es würde wohl schwerlich zu einer so dichten und massenhaften Entwickelung der Fadenpilze wie in unserem Falle gekommen sein; umgekehrt ist es bekannt, dass die Spaltpilze sich auf jeder freien Oberfläche einer in Zerfall begriffenen Substanz mit grosser Leichtigkeit niederlassen und zur 
Entwickelung gelangen. Ich glaube deshalb, dass in dem mitgetheilten Falle die eitrige Entzündung der Hornhant mindestens der Hauptsache nach durch Aspergilluswucherung hervorgerufen war.

Uebrigens möchte ich, soweit meine bisherigen Beob. achtungen mir ein Urtheil erlauben, die Keratomycosis aspergillina nur für ein seltenes und ansuahmsweises Vorkommniss halten, da ich seit jener Zeit in keinem anderen der ziemlich zahlreichen Fälle von Hypopyonkeratitis, welche ich beobachten konnte, wieder Aspergilluspilze in der Hornhaut gefunden habe.

Selbst in einem Falle, der mit ganz ähnlichen klinischen Erscheinungen, namentlich auch mit starker Chemosis ohne gleichzeitige erheblichere Betheiligung der Iris, einherging, fanden sich diese Pilze nicht, wohl aber schienen Microcokken vorhanden zu sein. Der Nachweis der letzteren in den kleinen necrotischen Stückchen, welche sich von dem Geschwürsgrunde abstossen, hat immer einige Schwierigkeiten, ist mir aber wiederholt in zweifelloser Weise gelungen, und ich glaube, dass man durch eine vollkommenere Untersuchungsmethode noch bessere Resultate erhalten würde.

In dem vorliegenden Falle spricht auch das ungewöhnlich ungünstige Resultat der Behandlung und der Ausgang in totales Lencom dafür, dass es sich hier um eine eigenthümliche und besondere Ursache handelte.

Nachdem ich übrigens in den Versuchen an Thieren gesehen habe, mit welcher Geschwindigkeit die Pilze sich in der Hornhaut weithin verbreiten und wie der von ihnen ergriffene Abschnitt regelmässig der Necrose und Abstossung durch reactive Eiterung anheimfällt, kann ich über den ungünstigen Ausgang des Leidens bei meinem Patienten durchaus nicht verwundert sein. 
Das von mir beobachtete Vorkommen einer durch Aspergilluswucherung erzeugten Hypopyonkeratitis ist sicher geeignet, die Annabme eines mycotischen Ursprunges der Entzündung auch in vielen anderen Fallen dieser deletären Hornhauterkrankung wesentlich $\mathrm{zu}$ unterstützen; auch glaube ich, dass die weitere Untersuchung der Vorgänge bei der ersteren uns noch manche Aufschlüsse über die Pathogenese der mycogenen Entzïndung im Allgemeinen liefern wird. 